\title{
Improved hospital safety performance and reduced medicolegal risk: an ecological study using 2 Canadian databases
}

\author{
Qian Yang MSc, Cathy Zhang MSc, Kristen Hines BA, Lisa A. Calder MD MSc
}

Abstract

Background: Few empirical studies have validated the relation between medicolegal risk and hospital patient safety performance. We sought to determine whether there was a relation between in-hospital patient safety events and medicolegal cases involving Canadian physicians.

Methods: In this ecological study, we used Poisson regression to compare data from the Canadian Institute for Health Information's Discharge Abstract Database and the database of the Canadian Medical Protective Association (CMPA) of medicolegal cases over 10 years (2005/06 to 2014/15). We identified incidents and cases based on 15 Agency for Healthcare Research and Quality patient safety indicators within the Canadian Institute for Health Information and CMPA data sets. We performed subgroup analyses for obstetrical and surgical cases.

Results: We found a statistically significant positive association between volume changes in patient safety indicator events $(n=$ $339741)$ and medicolegal cases $(n=15180)$ (parameter estimate 1.15, 95\% confidence interval [Cl] 0.4 to 1.9). This association suggests that, on average, a $10 \%$ decrease in events would correspond to a decrease of $11 \%$ in medicolegal cases. The degree of positive association varied by practice type, with obstetrics (97982 patient safety indicator events, 865 cases) showing a $25 \%$ decrease in medicolegal cases for every $10 \%$ decrease in events (parameter estimate $2.9,95 \% \mathrm{Cl} 0.5$ to 5.3 ) and surgery (168 886 patient safety indicator events, 4568 cases) showing a decrease of $9 \%$ for every $10 \%$ decrease in events (parameter estimate $0.9,95 \% \mathrm{Cl} 0.2$ to 1.7$)$.

Interpretation: The statistically significant positive association between patient safety indicator events and medicolegal cases quantifies a relation between patient safety and physician medicolegal risk in Canadian hospitals. This suggests new, practical uses for both medicolegal and patient safety indicator data in system-level quality-improvement efforts.

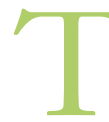
he number and scope of patient safety and qualityimprovement initiatives are growing, and these activities are increasingly being tied to incentives such as funding and accreditation. Because of their impact on hospital budgets and provider time, there is a pressing need to identify which safety or quality-improvement targets have the potential to produce the greatest benefits. At the same time, there is a drive to simplify the measures through which quality and safety are evaluated. Although administrative data including the Agency for Healthcare Research and Quality's patient safety indicators have been used to measure quality improvement and patient safety, their suitability for these purposes has been debated., ${ }^{1,2}$

In the field of patient safety, many studies have evaluated the impact of health care system improvements on medical liability, with much of this work focusing on obstetrics. ${ }^{3-12}$ Other research has attempted to study the link between the patient experience and various measures of quality and safety, with overall findings of a positive association between patient satisfaction, clinical effectiveness and patient safety. ${ }^{13}$ However, to date, few empirical studies have attempted to validate the existence of a relation between medicolegal risk and hospital patient safety performance, or to measure the effects of this association.

In 2010, the RAND Corporation's Institute for Civil Justice evaluated the relation between patient safety incidents and malpractice claims in California over a 5 -year period.

Competing interests: All of the authors are employed by the Canadian Medical Protective Association and have no competing interests to declare.

This article has been peer reviewed.

Correspondence to: Lisa Calder, Icalder@cmpa.org

CMAJ Open 2018. DOI:10.9778/cmajo.20180077 
That study used the Agency for Healthcare Research and Quality's patient safety indicators to identify patient safety incidents and showed a linear relation that represented a decrease of 3.7 malpractice claims for every 10 fewer incidents. It also confirmed that the correlation held true across multiple medical specialties. ${ }^{14}$ The objective of our study was to determine and quantify the relation between in-hospital patient safety events and medicolegal cases involving physicians in Canada.

\section{Methods}

\section{Setting, design and data sources}

In this ecological study, we used methods similar to those of the RAND study to analyze 10 years of data from the Canadian Institute for Health Information, an agency that collects and reports on data related to Canada's health care system, and the Canadian Medical Protective Association (CMPA), a nonprofit mutual defence organization that provides medicolegal advice and assistance to physicians. At the time of this study, over 93000 physicians were members of the CMPA (> 95\% of practising Canadian physicians).

We selected 15 patient safety indicators from the Agency for Healthcare Research and Quality's quality indicators ${ }^{15}$ that could be identified in Canadian hospital data (Table 1). We did not include "PSI 02 Death rate in low-mortality diagnosis related groups (DRGs)" or "PSI 04 Death rate among surgical inpatients with serious treatable conditions" as they could not be identified in the Canadian Institute for Health Information Discharge Abstract Database (DAD) owing to their reliance on diagnosis-related groups, which are not used as a classification system in Canada. We did not include "PSI 09 Perioperative hemorrhage or hematoma rate" because it could not be identified in the DAD owing to differences in the International Classification of Diseases (ICD) coding classification revisions used by the Agency for Healthcare Research and Quality and the Canadian Institute for Health Information for the study period. The agency's version 5 patient safety indicators were defined with the use of the clinical modification of the ICD, 9th revision, whereas the Canadian Institute for Health Information implemented the coding of the enhanced Canadian version of the 10 th revision of the International Statistical Classification of Diseases and Related Health Problems in 2005. We measured improvements in hospital patient safety performance by the annual changes in the frequency of these patient safety indicator events.

The DAD is a key database that captures administrative, clinical and demographic information on hospital discharges. It contains inpatient discharge data from all Canadian hospitals with the exception of those in the province of Quebec. The database records about 2.5 million inpatient hospital admissions each year. The CMPA maintains a national database that contains information on medicolegal cases involving Canadian physicians. Medicolegal cases are coded by a team of professionals with nursing or health information expertise who review the medical issues and legal information in each case. Medical coding follows national standards and analytical frameworks developed in house. To reduce the risk of misclassification, coders conduct weekly quality-assurance reviews of case coding. ${ }^{16}$

Table 1: Selected patient safety indicators reported in the Discharge Abstract Database, 2005/06 to 2014/15

\begin{tabular}{|c|c|c|c|}
\hline Patient safety indicator ${ }^{15}$ & $\begin{array}{c}\text { No. of events* } \\
n=339741\end{array}$ & \multicolumn{2}{|c|}{ Model } \\
\hline 03 Pressure ulcer event & 38136 & & \\
\hline 05 Retained surgical item or unretrieved device fragment events & 1979 & $\times$ & \\
\hline 06 latrogenic pneumothorax event & 21343 & & \\
\hline 08 Postoperative hip fracture event & 16848 & & \\
\hline 10 Postoperative physiologic and metabolic derangement event & 12009 & $\times$ & \\
\hline 11 Postoperative respiratory failure event & 5453 & $\times$ & \\
\hline 12 Perioperative pulmonary embolism or deep vein thrombosis event & 32066 & $x$ & \\
\hline 16 Transfusion reaction event & 168 & $x$ & \\
\hline 17 Birth trauma event - injury to neonate & 15827 & & $x$ \\
\hline 18,19 Obstetric trauma event - vaginal delivery with or without instrument & 82155 & & $x$ \\
\hline
\end{tabular}


For this study, we compared 10 years of data, from Apr. 1, 2005, to Mar. 31, 2015, from both databases. To be consistent with the data available to us through the $\mathrm{DAD}$, medicolegal cases that occurred in Quebec were excluded from the CMPA data set. Medicolegal case volume was represented by the frequency of all medicolegal cases that included not only civil legal actions but also medical regulatory authority and hospital complaints (Table 2). Because patients or their families may choose different pathways to bring a case forward, we elected to include all 3 case types to present a fuller picture of the overall medicolegal landscape.

The unit of analysis for both databases was event volume by region and by year. Event volume was represented by the number of patient safety indicator events in the DAD and the number of medicolegal cases in the CMPA database. We used event volume by region by year as the unit of analysis because our interest was in the overall volume rather than individual cases. British Columbia, Alberta and Ontario each formed a region on their own, and the 7 remaining provinces and the territories were combined into 1 region, labelled "the rest of Canada," for measurement stability. In determining regions, our goal was to make the measure as specific as possible while avoiding year-to-year fluctuations. The Prairie provinces, Atlantic provinces and territories were combined because of their relatively small populations, to create more appropriate comparisons based on population size.

For each region, we used the average number of medicolegal cases across the 10-year study period as the denominator and standardized the medicolegal case volume by calculating the annual ratio of the case count to the regional average. This standardized value of the medicolegal cases was the response variable in the regression models, as illustrated by the following equation:

Standard volume $e_{\text {rgion,year }}=$ annual frequency $_{\text {rggion,yean }} / 10$-year average rggion

Similarly, we standardized events from the DAD by region and by year. We used this standardized patient safety indicator event volume as the main predictor in the regression models. There were no built-in restrictions to the models; however, as described above, we combined the provinces with small populations and the territories to avoid extreme values

\begin{tabular}{|lccc|}
\hline $\begin{array}{l}\text { Table 2: Distribution of Canadian Medical Protective } \\
\text { Association cases used in modelling by type }\end{array}$ \\
\hline \multicolumn{4}{c|}{ Model; no. of cases } \\
\cline { 2 - 4 } Case type & $\begin{array}{c}\text { Overall } \\
n=15180\end{array}$ & $\begin{array}{c}\text { Obstetrical } \\
n=865\end{array}$ & $\begin{array}{c}\text { Surgical } \\
n=4568\end{array}$ \\
\hline Legal & 3639 & 521 & 1820 \\
\hline $\begin{array}{l}\text { Regulatory authority } \\
\text { complaint }\end{array}$ & 3757 & 270 & 1200 \\
\hline Hospital complaint & 7643 & 73 & 1527 \\
\hline Other* & 141 & 1 & 21 \\
\hline *Includes human rights complaints, inquests and privacy investigations. \\
\hline
\end{tabular}

caused by small denominators. We also treated all events equally since we were interested in overall volume and are unaware of any validated methods to weight points for this type of model.

Obstetrics and surgery are known to be unique practice types, such that they are both likely to see specific types of patient safety indicator events. Therefore, we built separate models for these subgroups to test for associations between patient safety indicator events and medicolegal cases. Within the same model, if a case from the DAD was involved in more than 1 patient safety indicator event, it was counted only once. When a case included events that were related to different models, the case was counted separately in the relevant models.

Although the purpose of this study was to examine the relation of hospital safety and medicolegal risk, there were no unique patient identifiers available to link the cases. As such, it was not possible to perform case-level data linkages between the $\mathrm{DAD}$ discharge records and medicolegal cases.

\section{Statistical analysis}

We used Poisson regression analysis with a log-linear link to test the significance of the association between volume changes for patient safety indicator events and medicolegal cases. To distribute expertise and reduce bias, 2 statisticians (Q.Y. and C.Z.) analyzed the data using SAS software v9.2 (SAS Institute). Considering the longitudinal nature of the data (i.e., the correlation of repeated measurements from the same region in different years), we used generalized estimating equations to address autocorrelation among responses. We included a set of year and region variables as well as their interaction terms to control for additional variations caused by temporal and regional difference in demographic charateristics, health care services and legal systems. The exponentiated parameter estimates for the patient safety indicator events were the expected effects of changing volume of patient safety indicator events on the volume of medicolegal cases. When the models showed statistical significance in these events, we plotted such effects using the parameter estimates to illustrate the association between the volume of patient safety indicator events and that of medicolegal cases.

\section{Ethics approval}

No patient information or patients were involved in this study; therefore, ethics approval was not necessary.

\section{Results}

We used 324592 distinct records from the DAD to construct the standardized values for the models. Because some of these records contained more than 1 patient safety indicator event, a total of 339741 patient safety indicator events were found in the records. From the CMPA database, we used 15180 medicolegal cases for the models (Table 2). Figure 1, A and $\mathrm{B}$ show the regional trends of medicolegal cases and the standardized ratios of annual case volume to regional average, respectively. The volume decreases seen in the CMPA data in the last few years of the study period can be explained by a lag 
A

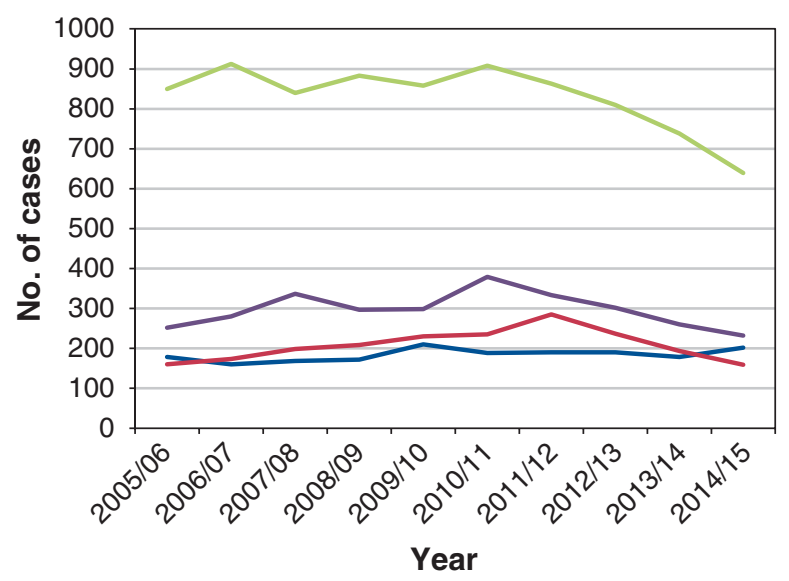

C

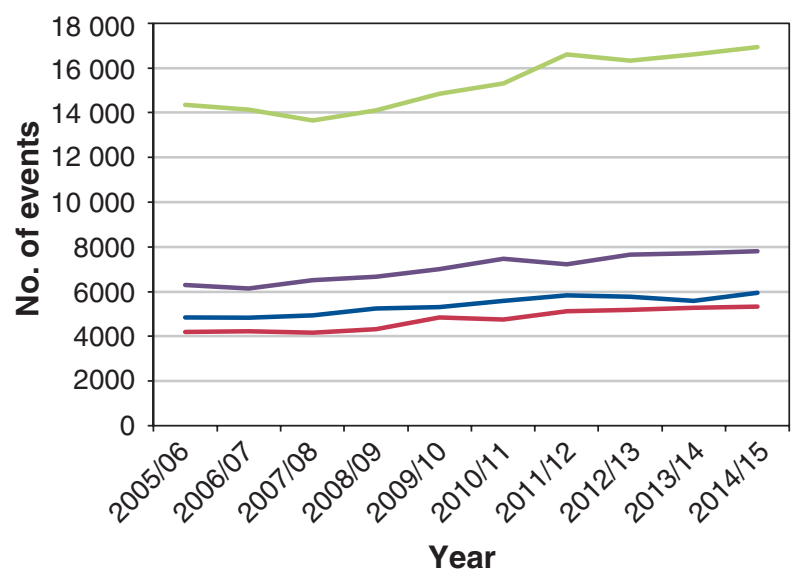

B

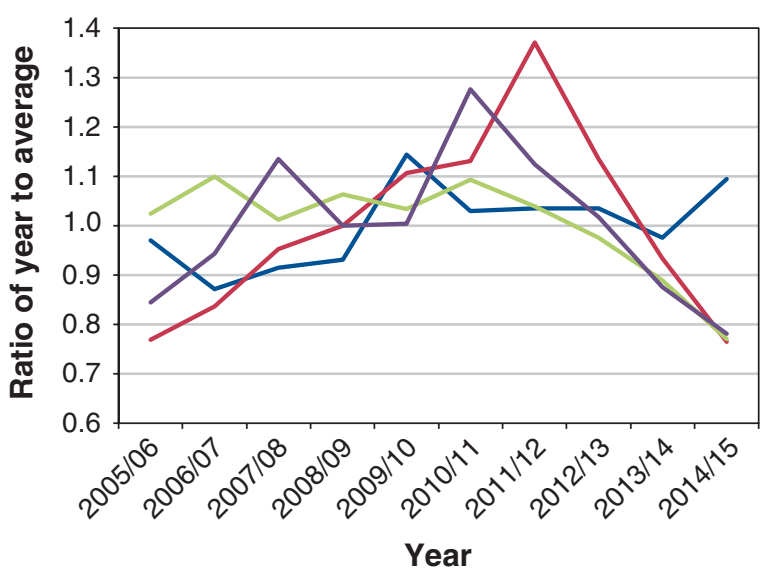

D

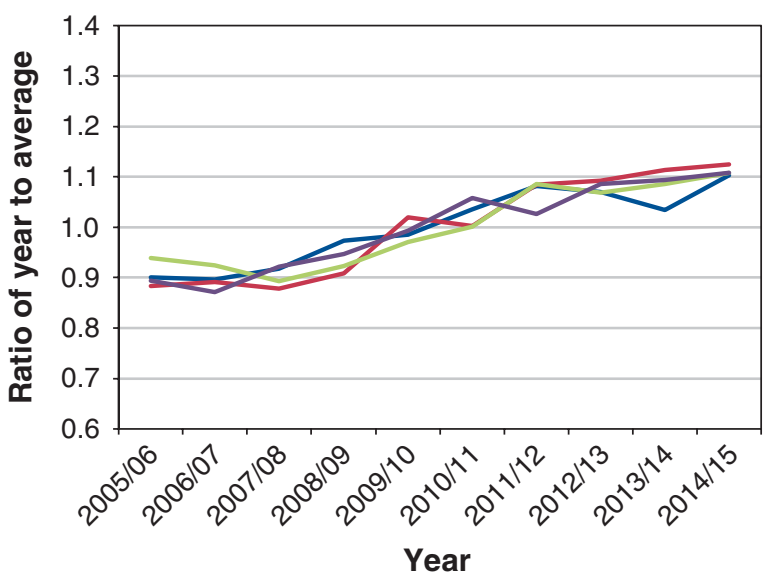

- Alberta - British Columbia - Ontario - Rest of Canada

Figure 1: Raw medicolegal case count (A) and standardized medicolegal case volume (B) by region, Canadian Medical Protective Association in-hospital cases; raw patient safety indicator event counts $(C)$ and standardized patient safety indicator event volume (D) by region, Discharge Abstract Database.

time that exists between when an event occurs and when a case is initiated. In general, about $50 \%$ of cases are reported within 2 years of the occurrence of the event, and $90 \%$ are reported within 4 years. To control for this time effect, we created a variable for year in the models to explain the artificial drop between event occurrence and medicolegal case initiation. As this artificial drop was not always the same across years (e.g., was not always a linear decrease, or the speed of decrease changed from year to year) and since year is a continuous interval variable, we also added a second-order term of year into the models at the beginning of the regression process. Figure 1, C and D show the regional trends of patient safety indicator events from the DAD and the standardized ratios of annual incident volume to regional average, respectively.

The modelling results are reported in Table 3 . The parameter estimate for the patient safety indicator event volume suggests a significant positive association $(1.15,95 \%$ con- fidence interval [CI] 0.4 to 1.9 ) between volume changes in patient safety indicator events and medicolegal cases when region and year are controlled for.

The annual ratio of patient safety indicator events varied between 0.9 and 1.1 (Figure 1, D). When the relation between patient safety indicator events and medicolegal cases was plotted within this range, applying the model results, it appeared to be roughly linear (Figure A1, Appendix 1, available at www.cmajopen.ca/content/6/4/E561/suppl/DC1). This projects that a region with a $10 \%$ increase in patient safety indicator events would see a corresponding increase of $12 \%$ in medicolegal cases, or, alternatively, with a $10 \%$ decrease in patient safety indicator events, an $11 \%$ decrease in medicolegal cases would be observed.

The obstetrical model analyzed 97982 patient safety indicator events identified in the DAD (or 97982 distinct DAD records that involved an obstetrical patient safety indicator 


\begin{tabular}{|c|c|c|c|}
\hline \multirow[b]{2}{*}{ Parameter } & \multicolumn{3}{|c|}{ Model; estimate $(95 \% \mathrm{Cl})$} \\
\hline & Overall & Obstetrical & Surgical \\
\hline Intercept & $-1.20(-2.00$ to -0.40$)$ & $-1.72(-3.74$ to 0.29$)$ & $-1.15(-1.85$ to -0.45$)$ \\
\hline $\begin{array}{l}\text { Patient safety indicator } \\
\text { event volume* }\end{array}$ & 1.15 (0.4 to 1.90$)$ & 2.91 (0.53 to 5.28$)$ & $0.92(0.17$ to 1.67$)$ \\
\hline \multicolumn{4}{|l|}{ Region } \\
\hline Alberta & $-0.15(-0.18$ to -0.13$)$ & $-0.43(-0.50$ to -0.37$)$ & $0.20(0.18$ to 0.21$)$ \\
\hline British Columbia & $-0.11(-0.12$ to -0.09 & $-0.61(-0.92$ to -0.31$)$ & $0.12(0.003$ to 0.24$)$ \\
\hline Ontario & 0.09 (0.08 to 0.09$)$ & $-0.23(-0.40$ to -0.07$)$ & $0.22(0.21$ to 0.23$)$ \\
\hline Rest of Canada & - & - & - \\
\hline Year & 0.09 (0.03 to 0.15$)$ & $-0.24(-0.31$ to -0.17$)$ & $0.22(0.17$ to 0.28$)$ \\
\hline Year $^{2} \dagger$ & $-0.01(-0.02$ to -0.004$)$ & - & $-0.03(-0.03$ to -0.02$)$ \\
\hline \multicolumn{4}{|l|}{ Region * year } \\
\hline Alberta * year & $0.03(0.02$ to 0.03$)$ & 0.10 (0.08 to 0.11$)$ & $-0.04(-0.04$ to -0.03$)$ \\
\hline British Columbia * year & 0.02 (0.02 to 0.02$)$ & $0.12(0.06$ to 0.18$)$ & $-0.02(-0.04$ to -0.001$)$ \\
\hline Ontario * year & $-0.02(-0.02$ to -0.01$)$ & 0.05 (0.02 to 0.09$)$ & $-0.04(-0.05$ to -0.04$)$ \\
\hline Rest of Canada * year & - & - & - \\
\hline \multicolumn{4}{|c|}{$\begin{array}{l}\text { Note: } \mathrm{Cl}=\text { confidence interval. } \\
{ }^{*} \text { These parameter estimates can alternatively be depicted as equations: } \\
\text { Log }(\text { medicolegal case volume })=1.15^{*} \text { patient safety indicator event volume }+(\text { intercept }+ \text { control variables) for the overall model, } \\
\text { Log (medicolegal case volume })=2.91{ }^{*} \text { patient safety indicator event volume }+(\text { intercept }+ \text { control variables) for the obstetrical } \\
\text { model and Log }(\text { medicolegal case volume })=0.92{ }^{*} \text { patient safety indicator event volume }+(\text { intercept }+ \text { control variables) for the } \\
\text { surgical model. } \\
\text { †The higher-order year variable }\left(y e a r^{2}\right) \text { was not significant and was thus removed from the final model. }\end{array}$} \\
\hline
\end{tabular}

event) and 865 obstetrics-related medicolegal cases. The surgical model analyzed 168886 patient safety indicator events from the DAD (or 160598 distinct DAD records that involved at least 1 surgical patient safety indicator event) and 4568 medicolegal cases. We calculated regional annual change ratios for both patient safety indicator events and medicolegal cases in the same way as the all-cases model for both specialty models. The obstetrics model showed a $25 \%$ decrease in medicolegal cases for every $10 \%$ decrease in events; the surgical model showed a decrease of $9 \%$ for every $10 \%$ decrease in events (Figures A2 and A3, Appendix 1).

Obstetrical medicolegal cases showed the greatest rate reduction when patient safety indicator events decreased. The parameter estimate for obstetrical case modelling suggests a significant positive association $(2.9,95 \%$ CI 0.5 to 5.3$)$ (Table 3 ). This projects that a region with a $10 \%$ decrease in patient safety indicator events would see a corresponding decrease of $25 \%$ in medicolegal cases. However, the degree of increase in medicolegal case volume is far greater than that of the patient safety indicator event, with an average increase of $34 \%$ for every $10 \%$ increase of patient safety indicator. The parameter estimate for surgical case modelling also suggests a significant positive association $(0.9,95 \%$ CI 0.2 to 1.7$)$ (Table 3$)$. This projects that a region with a $10 \%$ increase in patient safety indicator events would see a corresponding increase of $10 \%$ in medicolegal cases, or, alternatively, with a decrease of $10 \%$ in patient safety indicator events, a $9 \%$ decrease in medicolegal cases would be observed.

\section{Interpretation}

This comparison of 2 Canadian databases suggests a relation between hospital safety performance and medicolegal risk in Canada. The degree of positive association varied by practice type, with obstetrics showing a stronger relation. This association may reflect the severity of events encompassed by patient safety indicator 17 , which include neurologic injury to the infant. These types of injuries are a major source of malpractice litigation for obstetricians given the vulnerability of patients and lifelong care costs associated with these events.

This study joins a small body of research that has shown relations between patient safety indicator events and increased medicolegal risk, ${ }^{14}$ lower safety culture scores ${ }^{17}$ and patient ratings of physicians and nurses ${ }^{18}$ and poorer performance on other measures of safety and quality. ${ }^{19,20}$ Our results are consistent with those of studies that have shown that improved safety and quality are associated with decreased medicolegal risk. ${ }^{3-12}$

At the system level, longitudinal trends in medicolegal data can be used to clearly illustrate the institutional impact of quality-improvement and patient safety initiatives for front-line providers. Therefore, our results may support efforts to position patient safety at the forefront of medical malpractice reform by showing that reduced medicolegal liability could be a secondary benefit of improved patient safety. Although researchers have questioned the use of patient safety indicators and other administrative data sources for quality-improvement 
purposes on the basis of inadequate reliability ${ }^{1}$ and clinical relevance, ${ }^{2}$ our results suggest that the current set of indicators may represent a more consistent measure of patient safety than previously argued.

\section{Limitations}

Because the patient safety indicators are acute care related, they do not reflect the full spectrum of clinical care. Also, whereas the DAD includes all inpatient health event data, the CMPA data include only cases that involved a physician. It is important to note that, although the CMPA represents most physicians in Canada, members report hospital and regulatory authority complaints to the CMPA at their own discretion, and therefore these cases do not represent a complete picture of all such cases in Canada. Furthermore, to ensure comparability between data sets, medicolegal cases from the province of Quebec were not included in this analysis, as data from that province were not available from the Canadian Institute for Health Information despite repeated attempts to obtain them. Therefore, our results do not represent a complete picture of Canada.

Samples for medicolegal cases were relatively small for obstetrical cases at the regional level, and the confidence intervals for the parameter estimates were wide. This is explained in part by the fact that because obstetrical cases often concern patients of minority age, they have longer limitation periods (generally when the plaintiff reaches age $18 \mathrm{yr}$ plus $2 \mathrm{yr}$ ), which results in longer lag times between event occurrence and medicolegal case initiation. Complaints to hospitals and regulatory authorities represented a substantial portion of the medicolegal cases included in this analysis. The characteristics of these types of cases may differ from those of legal actions. For example, whereas the majority of legal actions relate to allegations of harm, hospital and regulatory authority complaints often involve communication, manner and administrative issues. It should be noted that even cases not directly associated with harm from health care delivery may reflect important elements of patient safety culture.

\section{Conclusion}

Although the reasons why medicolegal cases arise are often complex and multifactorial, the statistically significant positive association between patient safety indicator events and medicolegal cases quantifies a relation between patient safety and medicolegal risk in Canadian hospitals. This finding challenges critiques about the suitability of the Agency for Healthcare Research and Quality's patient safety indicators as measures of safety and quality and suggests new, practical uses for both medicolegal and patient safety indicator data in system-level quality-improvement efforts.

\section{References}

1. Winters BD, Bharmal A, Wilson RF, et al. Validity of the Agency for Healthcare Research Quality patient safety indicators and the Centers for Medicare and Medicaid hospital-acquired conditions. Med Care 2016;54:1105-11.

2. Kachalia A, Mello MM, Nallamothu BK, et al. Legal and policy interventions to improve patient safety. Circulation 2016;133:661-71.

3. Clark SL, Belfort MA, Byrum SL, et al. Improved outcomes, fewer cesarean deliveries, and reduced litigation: results of a new paradigm in patient safety. Am 7 Obstet Gynecol 2008;199:105.e1-7.
4. Grunebaum A, Chervenak F, Skupski D. Effect of a comprehensive obstetric patient safety program on compensation payments and sentinel events. $A m \mathcal{F}$ Obstet Gynecol 2011;204:97-105.

5. Howell AM, Burns EM, Bouras G, et al. Can patient safety incident reports be used to compare hospital safety? Results from a quantitative analysis of the English national Reporting and Learning System data. PLoS One 2015;10: e0144107.

6. Illingworth KD, Shaha SH, Tzeng TH, et al. The impact of tort reform and quality improvements on medical liability claims: a tale of 2 states. $\mathrm{Am} 7 \mathrm{Med}$ Qual 2015;30:263-70.

7. Iverson RE Jr, Heffner LJ. Patient safety series: obstetric safety improvement and its reflection in reserved claims. Am f Obstet Gynecol 2011;205:398-401.

8. Milne JK, Lalonde AB. Patient safety in women's health-care: professional colleges can make a difference. The Society of Obstetricians and Gynaecologists of Canada MORE(OB) program. Best Pract Res Clin Obstet Gynaecol 2007;21:565-79.

9. Pegalis SE, Bal BS. Closed medical negligence claims can drive patient safety and reduce litigation. Clin Orthop Relat Res 2012;470:1398-404.

10. Pettker CM, Thung SF, Lipkind HS, et al. A comprehensive obstetric patient safety program reduces liability claims and payments. Am $\mathcal{F}$ Obstet Gynecol 2014; 211:319-25.

11. Riley W, Meredith LW, Price R, et al. Decreasing malpractice claims by reducing preventable perinatal harm. Health Serv Res 2016;51(Suppl 3): 2453-71.

12. Simpson KR, Kortz CC, Knox E. A comprehensive perinatal patient safety program to reduce preventable adverse outcomes and costs of liability claims. 7t Comm 7 Qual Patient Saf 2009;35:565-74.

13. Doyle C, Lennox L, Bell D. A systematic review of evidence on the links between patient experience and clinical safety and effectiveness. BM7 Open 2013;3:e01570.

14. Greenberg MD, Haviland AM, Ashwood JS, et al. Is better patient safety associated with less malpractice activity? Evidence from California [technical report]. Santa Monica (CA): RAND Corporation; 2010.

15. AHRQ Quality Indicators ${ }^{\mathrm{TM}}$ (AHRQ QI ${ }^{\mathrm{TM}}$ ) ICD-9-CM and ICD-10-CM/PCS specification enhanced version 5.0: Inpatient Quality Indicators \#16 (IQI \#16) beart failure mortality rate. Rockville (MD): Agency for Healthcare Research and Quality, US Department of Health and Human Services; 2015.

16. McCleery A, Devenny K, Ogilby C, et al. Using medicolegal data to support safe medical care: a contributing factor coding framework. 7 Healthc Risk Manag 2018 Aug. 3 [Epub ahead of print]. doi: 10.1002/jhrm.21348.

17. Mardon RE, Khanna K, Sorra J, et al. Exploring relationships between hospital patient safety culture and adverse events. F Patient Saf 2010;6:226-32.

18. Kemp KA, Santana MJ, Southern DA, et al. Association of inpatient hospital experience with patient safety indicators: a cross-sectional Canadian study. BM7 Open 2016;6:e011242.

19. Gray DM 2nd, Hefner JL, Nguyen MC, et al. The link between clinically validated patient safety indicators and clinical outcomes. Am 7 Med Qual 2017;32: 583-90.

20. Rosen AK, Rivard P, Zhao S, et al. Evaluating the patient safety indicators: How well do they perform on veterans health administration data? Med Care $2005 ; 43: 873-84$.

Affiliations: Canadian Medical Protective Association (Yang, Zhang, Hines, Calder); Ottawa Hospital Research Institute (Calder); Department of Emergency Medicine (Calder), University of Ottawa, Ottawa, Ont.

Contributors: Qian Yang conceived the study and contributed to the study design and to drafting the manuscript. Cathy Zhang contributed to the study design and to drafting the manuscript, and analyzed the data under the supervision of Qian Yang. Kristen Hines contributed to data interpretation and to drafting the manuscript. Lisa Calder reviewed the study design and contributed to data interpretation. All of the authors critically revised the manuscript for important intellectual content, gave final approval of the version to be published and agreed to be accountable for all aspects of the work.

Acknowledgements: The authors thank Allan McDougall for providing editorial support for the manuscript and Ria De Gorter for supporting the manuscript submission process.

Previous presentation: These results were presented at the 2016 annual conference of the Canadian Association for Health Services and Policy Research, Toronto, May 9-12, 2016.

Disclaimer: Parts of this material are based on data and information provided by the Canadian Institute for Health Information (CIHI). However, the analyses, conclusions, opinions and statements expressed herein are those of the authors and not necessarily those of the CIHI.

Supplemental information: For reviewer comments and the original submission of this manuscript, please see www.cmajopen.ca/content/6/4/ E561/suppl/DC1. 